\title{
Mapping and functional characterization of the murine Smoothelin-like 1 promoter
}

\author{
Annegret Ulke-Lemée, Sara R Turner, Saad H Mughal, Meredith A Borman, Robert J Winkfein, Justin A MacDonald
}

\begin{abstract}
Background: Smoothelin-like 1 (SMTNL1, also known as CHASM) plays a role in promoting relaxation as well as adaptive responses to exercise, pregnancy and sexual development in smooth and skeletal muscle. Investigations of Smtn/1 transcriptional regulation are still lacking. Thus, in this study, we identify and characterize key regulatory elements of the mouse Smtn/1 gene.

Results: We mapped the key regulatory elements of the Smtn/1 promoter region: the transcriptional start site (TSS) lays -44 bp from the translational start codon and a TATA-box motif at -75 bp was conserved amongst all mammalian Smtn/1 promoters investigated. The Smtn/1 proximal promoter enhances expression up to 8-fold in smooth muscle cells and a second activating region lays $500 \mathrm{bp}$ further upstream. Two repressing motifs were present (-118 to $-218 \mathrm{bp}$ and -1637 to $-1869 \mathrm{bp}$ ). The proximal promoter is highly conserved in mammals and contains a mirror repeat sequence. In silico analysis suggests many transcription factors (notably MyoD) could potentially bind within the Smtn/1 proximal promoter sequence.

Conclusion: $\mathrm{Smtn} / 1$ transcript was identified in all smooth muscle tissues examined to date, albeit at much lower levels than found in skeletal muscle. It is unlikely that multiple SMTNL1 isoforms exist since a single Smtn/1 transcription start site was identified in both skeletal and intestinal smooth muscle. Promoter studies suggest restrictive control of $5 \mathrm{mtn} / 1$ expression in non-muscle cells.
\end{abstract}

\section{Background}

The smoothelin-like 1 (SMTNL1 [Swiss-Prot: Q99LM3]) protein was discovered as a novel protein phosphorylated in response to cGMP stimulation of ileal smooth muscle tissue [1]. The protein contains a calponin homology domain at its carboxy-terminus, thus it was originally termed the calponin homology-associated smooth muscle protein (CHASM). Experiments completed in situ with isolated smooth muscle tissues suggest a physiological role for SMTNL1 in promoting the relaxant actions of PKA/PKG [1,2], and studies with Smtnl1 genetic knock-out mice link SMTNL1 with adaptive responses to exercise in both smooth and skeletal muscle [3]. More recent studies have provided indications that SMTNL1 also governs smooth and skeletal muscle adaptations during sexual development and pregnancy [4]. Although less well studied at the

\footnotetext{
* Correspondence: jmacdo@ucalgary.ca

* Correspondence: jmacdo@ucalgary.ca
Smooth Muscle Research Group and Department of Biochemistry \& Molecular Biology, University of Calgary, 3280 Hospital Drive NW, Calgary, Alberta, T2N 4Z6, Canada
}

(c) 2011 Ulke-Lemée et al; licensee BioMed Central Ltd. This is an Open Access article distributed under the terms of the Creative Commons Attribution License (http://creativecommons.org/licenses/by/2.0), which permits unrestricted use, distribution, and reproduction in any medium, provided the original work is properly cited. molecular level, current data suggests SMTNL1 plays an and development. The protein is known to interact with tropomyosin [5] and with apo-calmodulin in a $\mathrm{Ca}^{2+}$ dependent manner [6], to inhibit myosin phosphatase activity $[2,3]$ and to regulate the expression of the myosin phosphatase targeting subunit (MYPT1) [4].

As its name suggests, SMTNL1 is closely related to the smoothelin family of proteins (SMTN) that are used as markers for contractile smooth muscle cell differentiation $[7,8]$. The specific biological role of the two SMTN isoforms, A (short isoform, predominantly visceral expression) and B (long isoform, predominantly vascular expression), remains poorly defined; however, the analysis of mice lacking SMTN-A or -B has revealed critical roles for each of the proteins in intestinal and vascular smooth muscle performance, respectively $[9,10]$. Interestingly, the SMTN-A and SMTN-B isoforms are expressed from alternative promoters, with the intragenic promoter of SMTNA residing within exon 10 of the Smtn gene [11]. Thus, one Smtn gene gives rise to both SMTN-A and SMTN-B 
mRNA with lengths of 1700 and $3000 \mathrm{nt}$, respectively. The $S m t n$ promoter is controlled by serum response factor (SRF) and myocardin [12]. SRF and myocardin play critical roles in the expression and regulation of growth-responsive genes as well as the expression of virtually all smooth muscle specific genes, such as calponin, myosin heavy chain, $\alpha$-actin and SM-22 $[13,14]$. Previous analyses of various smooth muscle and general tissues by immunohistochemistry and Western blotting have revealed strong expression of SMTNL1 in MHC-2A skeletal muscle fibers, moderate expression in smooth muscle tissues (e.g., bladder, ileum, uterus and aorta) and no expression in MHC-I/ b cardiac muscle [3]. Given that SMTNL1 is expressed in multiple muscle types, it was expected that Smtnl1 transcriptional regulation might differ from the other smoothelin family members.

To date, the contribution of the SMTNL1 protein in smooth muscle contraction has been examined in vitro and in vivo [1-6], but investigations of its gene and transcriptional regulation are still lacking. Thus, in this study, we identify and characterize key regulatory elements of the promoter region in the mouse Smtnl1 gene. This region contains a TSS mapped to a location 119 bp downstream of the NCBI-predicted site. Our data demonstrate that a proximal promoter region is located within $118 \mathrm{bp}$ of the TSS site and provide molecular insights into the regulation of the Smtnl1 gene.

\section{Results and Discussion}

\section{PCR analysis of Smtnl1 transcript}

We first examined the pattern of Smtnl1 expression in skeletal muscle and representative smooth muscle tissues with RT-PCR. As shown in Figure 1A, the Smtnl1 transcript is expressed at very low levels. Smtnl1 expression in skeletal and aortic smooth muscle tissues was detectable following 35-cycles of amplification; however, transcript was not detected by RT-PCR in other smooth muscle beds. To increase the sensitivity of detection, we then performed nested PCR reactions on the primary products. This step also increased the specificity of the PCR reaction and permitted qualitative verification of Smtnl1 expression. We detected Smtnl1 expression by nested PCR in the muscle tissues selected for analysis. The nested-PCR amplicons were sequenced and align $100 \%$ to the Smtnl1 sequence. Due to its low abundance, we were unable to accurately assess the expression levels in different tissues with qPCR techniques.

We also investigated the expression of mRNA encoding SMTNL1 transcripts in various human tissues with Northern blot analysis. For this study, we used a 1.0-kb 5'-probe based on mouse Smtnl1 and moderate stringency washes. The probe was generated to include the sequence encoding the $\mathrm{N}$-terminal 273 amino acid residues of SMTNL1 in order to avoid cross-reactivity with
A

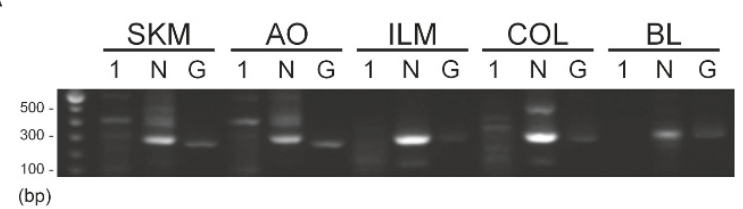

B

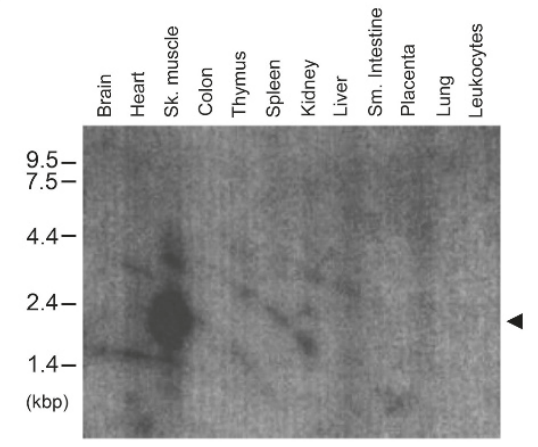

Figure 1 Expression profile of $S m t n / 1$ in various mouse and human tissues. In (A), an RT-PCR reaction (denoted $\mathbf{1}$; expected size, $440 \mathrm{bp}$ ) was performed on total RNA extracted from mouse skeletal muscle (SKM) and smooth muscle dissected from aorta $(\mathrm{AO})$, ileum (ILM), colon (COL) and urinary bladder (BL). The RT-PCR was followed by nested PCR (denoted $\mathbf{N}$; expected size, $313 \mathrm{bp}$ ) to increase the specificity and sensitivity of the detection. RT-PCR reactions of GAPDH (denoted $\mathbf{G}$; expected size, 240 bp) was performed to control for the integrity of the extracted mRNA. Results are representative of replicate PCR reactions completed on cDNA synthesized from two separate mice. In (B), Northern blot analysis of Smtn/1 expression in human tissues was completed on a Clontech multi-tissue membrane with $1 \mu \mathrm{g}$ polyA+ RNA loaded per lane. The membrane was hybridized overnight with a ${ }^{32} \mathrm{P}$-labeled Smtnl1 cDNA probe (1 - 1038 bp) and exposed to X-ray film. The major human $\mathrm{Smtn} / 1$ transcript of $2.1-\mathrm{kb}$ is indicated by the arrowhead.

other transcripts that possess sequence encoding a calponin homology $(\mathrm{CH})$-domain, such as the smoothelins. A single transcript of $\sim 2.1-\mathrm{kb}$ was detected in skeletal muscle (Figure 1B) and correlated with the predicted mRNA size of $1.8-\mathrm{kb}$. No other mRNA species were identified, implying that a single $S m t n l 1$ transcript exists in skeletal muscle. Although we obtained a strong signal for Smtnl1 mRNA in skeletal muscle, northern signals were not detected in tissues with high vascular content such as brain and kidney or in tissues with high smooth muscle content (e.g., placenta, small intestine or colon). As seen from our nested-PCR results, a number of smooth muscle tissues do possess Smtnl1 mRNA, but at much lower levels than skeletal muscle which might account for the inability to detect expression by Northern blotting. These data agree with protein distributions as determined previously by Western blotting [3]. Interestingly, recent studies have revealed that exercise- and 
pregnancy-induced alterations in SMTNL1 expression were linked to functional adaptations in skeletal and smooth muscle contractile performances. Given these previous findings, it will be important to generate an accurate tool to quantitatively analyze Smtnl1 transcript levels in human tissues and/or mouse models of disease.

\section{Smtnl1 transcription start site mapping}

The murine Smtnl1 gene [GenBank: 68678] is located on chromosome 2, E1 and spans 11.48-kb with 8 exons. The mRNA sequence [GenBank: NM_024230] has been annotated using bioinformatic and curatorial analyses in the NCBI Refseq database. A thymidine residue at -162 bp from the translational start site (AUG) within the Smtnl1 mRNA was assigned as the provisional transcriptional start site (TSS). This positions the ATG at the start of exon 2 while exon 1 (162 nt) is not translated. To verify the location of the TSS in the Smtnl1 gene, we performed 5'-RACE experiments on murine smooth and skeletal total RNA. Three reverse, gene-specific primers were designed for the PCR step that follows cDNA synthesis in the 5-RACE process (see Table 1). Primer GSP3 and GSP 2 anneal just downstream of the TSS and upstream of the AUG (expected PCR product $63 \mathrm{bp}$ and $176 \mathrm{bp}$, respectively). Primer GSP1 anneals just upstream and outside of the $\mathrm{CH}$ domain in exon 8 (expected PCR product $1479 \mathrm{bp}$ ).

The 5'-RACE PCR on smooth muscle (Figure 2A) and skeletal muscle (Figure 2B) total RNA produced low (< $250 \mathrm{bp}$ ) molecular weight cross-dimer products between the GSP and 5'-RACE UPM primers (as verified by sequencing). When skeletal muscle mRNA was used as template together with primer GSP1, a faint band was visible at approximately $1.5-\mathrm{kb}$ that was close to the expected product size (Figure 2B). To increase specificity and sensitivity, a second round of PCR was performed using nested primers on the products from the 5'-RACE PCR. The expected amplicon sizes with the NCBI-predicted TSS were 63 bp for NGSP1 and 823 bp for NGSP2. A strong band of approximately $700 \mathrm{bp}$ was observed for nested PCR reactions completed on both smooth and skeletal muscle GSP1 PCR products (Figure 2A \&2B), somewhat smaller than the expected $823 \mathrm{bp}$ product for the NGSP2 primer. Sequence analysis revealed a partial alignment of the 823 bp NGSP2 product with Smtnl1, starting within exon 1.

The 5 ' end of the product was 119 bp shorter than expected from the NCBI-predicted TSS. To further validate the TSS identified with 5'-RACE, ribonuclease protection assays (RPA) were performed. A ssRNA riboprobe (511 nt) was generated to span both the NCBIpredicted and 5'-RACE-derived TSS. A protected fragment of $369 \mathrm{bp}$ was expected for the NCBI-predicted TSS whereas a 231 bp fragment was expected for the newly derived TSS. As shown in Figure 2C, RPA was completed with smooth and skeletal muscle mRNA and biotin-18-UTP-labeled riboprobe. As expected, the fulllength probe was detected near the 500 bp marker. A protected fragment slightly larger than $200 \mathrm{bp}$ was detected in the RPA with the addition of skeletal muscle mRNA. This result further confirms the TSS location to be different from that annotated in the NCBI database, occurring less than $50 \mathrm{nt}$ upstream of the translational start codon. No protected fragment was detected when yeast RNA (control) or smooth muscle RNA was used in the RPA. The latter result was likely due to lack of sensitivity of the RPA conditions for low abundance mRNAs.

Analysis of the sequence located upstream of the start codon (ATG) in murine Smtnl1 indicated the presence of an initiator (Inr) sequence and a TATA box (Figure 2D). Therefore, our experimentally mapped Smtnl1 TSS corresponded to a thymidine within the Inr sequence (CTAGAAC, where the $\mathrm{T}$ was the first transcribed nucleotide) and was located 44 nt upstream of the translational start codon. The putative TATA box sequence was identified at -23 to -31 nt upstream of the new TSS. Further sequence analysis showed that the Inr and TATA sequences of Smtnll are conserved within a variety of mammalian Smtnl1 genes.

The expression profile of Smtnl1 differs from the other smoothelin (Smtn) family members that are exclusively expressed in differentiated smooth muscle cells. The Smtnl1 promoter was expected to be different since the smoothelins are not expressed in any tissue but smooth muscle cells. Indeed, the promoter sequences of smoothelins and Smtnl1 do not bear any resemblance to each other, despite some similarities in the protein-coding region (mainly within the $\mathrm{C}$-terminal calponin homology domain). The SMTN-A and SMTN-B isoforms of the Simtn gene are generated by alternative transcriptional start sites [11] that provide selective expression of each isoform in visceral and vascular smooth muscle beds, respectively. Moreover, alternative splicing produces three different $\mathrm{C}$-terminal variants of each SMTN isoform [15]. It is unlikely that multiple SMTNL1 isoforms exist since a single Smtnl1 TSS was identified from both skeletal and intestinal smooth muscle total RNA with sequencing of several 5'-RACE clones. All sequences commenced at a thymine located $44 \mathrm{nt}$ upstream of the translational start site (ATG). However, we have not yet completed 5'-RACE on Smtnl1 transcripts isolated from vascular tissue. Furthermore, we cannot exclude the presence of multiple C-terminal splice variants since we did not complete 3'-RACE analysis.

\section{SMTNL1 promoter reporter assay}

To locate cis-elements in the core promoter region that modulate expression of Smtnl1, we constructed deletion 
Table 1 PCR primers used in the analysis of Smtnl1

\begin{tabular}{|c|c|}
\hline \multicolumn{2}{|l|}{ RT-PCR \& nested PCR } \\
\hline mSmtn|1-F1 & AAGAAGGATCGAGCACCAGAAC \\
\hline mSmtn|1-R1 & ACACACTTGGAATCGGGCAC \\
\hline mSmtnl1-nested-F1 & GGAAGGCCATCATGGACAAATT \\
\hline mSmtnl1-nested-R1 & GCACAGTCGGCTAGTITCTCTG \\
\hline \multicolumn{2}{|l|}{ 5'-RACE } \\
\hline $5^{\prime}-\mathrm{CDS}$ & $\mathrm{dT}_{25} \mathrm{VN}(\mathrm{N}=\mathrm{A}, \mathrm{C}, \mathrm{G}$, or $\mathrm{T} ; \mathrm{V}=\mathrm{A}, \mathrm{G}$, or $\mathrm{C})$ \\
\hline SMART ॥ A & AAGCAGTGGTATCAACGCAGAGTACGCGGG \\
\hline UPM & $\begin{array}{l}\text { 5:1 mixture of (1): (2) } \\
\text { (1) CTAATACGACTCACTATAGCAAGCAGTGGTATCAACGCAGAGT } \\
\text { (2) CTAATACGACTCACTATAGC }\end{array}$ \\
\hline NUPM & AAGCAGTGGTATCAACGCAGAGT \\
\hline GSP1 & CCACCTCCAGCAGCTGGGCACAGTCGGCTAG \\
\hline GSP2 & GCCTCGTTCTAGCCGAGCTGCCTCACTCTCCAT \\
\hline GSP3 & CACCCCACCTTCAAGAGGCCCGGGCTTCG \\
\hline NGSP1 & CACCCCACCTTCAAGAGGC \\
\hline NGSP2 & CCAACTCCTTTGTGTCACTTCCG \\
\hline \multicolumn{2}{|l|}{ RPA } \\
\hline RPA2 & $\begin{array}{l}\text { TAATACGACTCACTATAGGGCTGATCAGATGCTAGGTACACTT } \\
\text { GCTTCCACCACCCCTTGCTTCCACC }\end{array}$ \\
\hline T7 promoter & TAATACGACTCACTATAGGG \\
\hline RPA-NCS & GAGAGGGACGCAGCAGCGTG \\
\hline RPA-NCA & GGTTCTGCCTGCAGCTCGG \\
\hline RPA-M1-2S & TAGAACGAGGCAAGAGCTCC \\
\hline RPA-M1-2A & GTTCTGCCCAATCTGAAGGC \\
\hline RPA-FR1 & GCCACCTGTCAGATCTTCGG \\
\hline RPA-RT7 & $\begin{array}{l}\text { TAATACGACTCACTATAGGGCTGATCAGATGCTAGGTACAG } \\
\text { TTCTGCCCAATCTGAAG }\end{array}$ \\
\hline
\end{tabular}

Promoter Truncations

\begin{tabular}{ll}
\hline+100 bp LUC R1 & GTGGGATCCGTGAAAGGTAAGAATAGG \\
0 bp LUC F1 & CTAGCCTTCTAGAACGAGGCAAGAGCTCC \\
-118 bp LUC F2 & CTAGAATCCTTCCCGAGCCCGGGC \\
-218 bp LUC F3 & CTCGATTCGCAGCGTGATTGAAAGATG \\
-468 bp LUC F4 & CTAGAATTCCCCCTCAGCAGCTACTTTG \\
-767 bp LUC F5 & CTCGATTCCGTTGGGACTGGGAATTCCG \\
-1350 bp LUC F6 & CTAGAATTCGACAAAGCTTGGGTCTATATC \\
-1637 bp LUC F7 & CTAGAATCCTCTTAACCTCCAAGACATC \\
-1869 bp LUC F8 & CTAGAATCCTCTTAACCTCCAAGACATC \\
-2368 bp LUC F9 & CTAGAATCCAAAGTCCTATTGCTTGCACC \\
-2759 bp LUC F10 & GCTGAATCGAATATCTCATGGACTTGCCTAGAG
\end{tabular}

mutants of the Smtnl1 upstream sequence and monitored Gaussia luciferase reporter activities in rat aortic A7r5 smooth muscle cells. Promoter sequences were generated spanning up to $2.7-\mathrm{kb}$ upstream of the newly derived Smtnl1 TSS and cloned into a promoterless pGLuc luciferase vector. Figure 3A shows the relative luciferase activity using progressively shorter promoter constructs. A 3.5-fold induction was observed for the upstream region spanning +100 to -2759 . Deletion of the region from -1637 to -1869 increased the luciferase induction to 8.5 -fold while removal of the sequence from -468 to -1637 elicited no further effect. The minimal sequence establishing the proximal promoter ( 0 to -118) provided a 7 -fold luciferase induction. Finally, the first 100 bases downstream of the new TSS had no activity. These data reveal two regions of promoter 


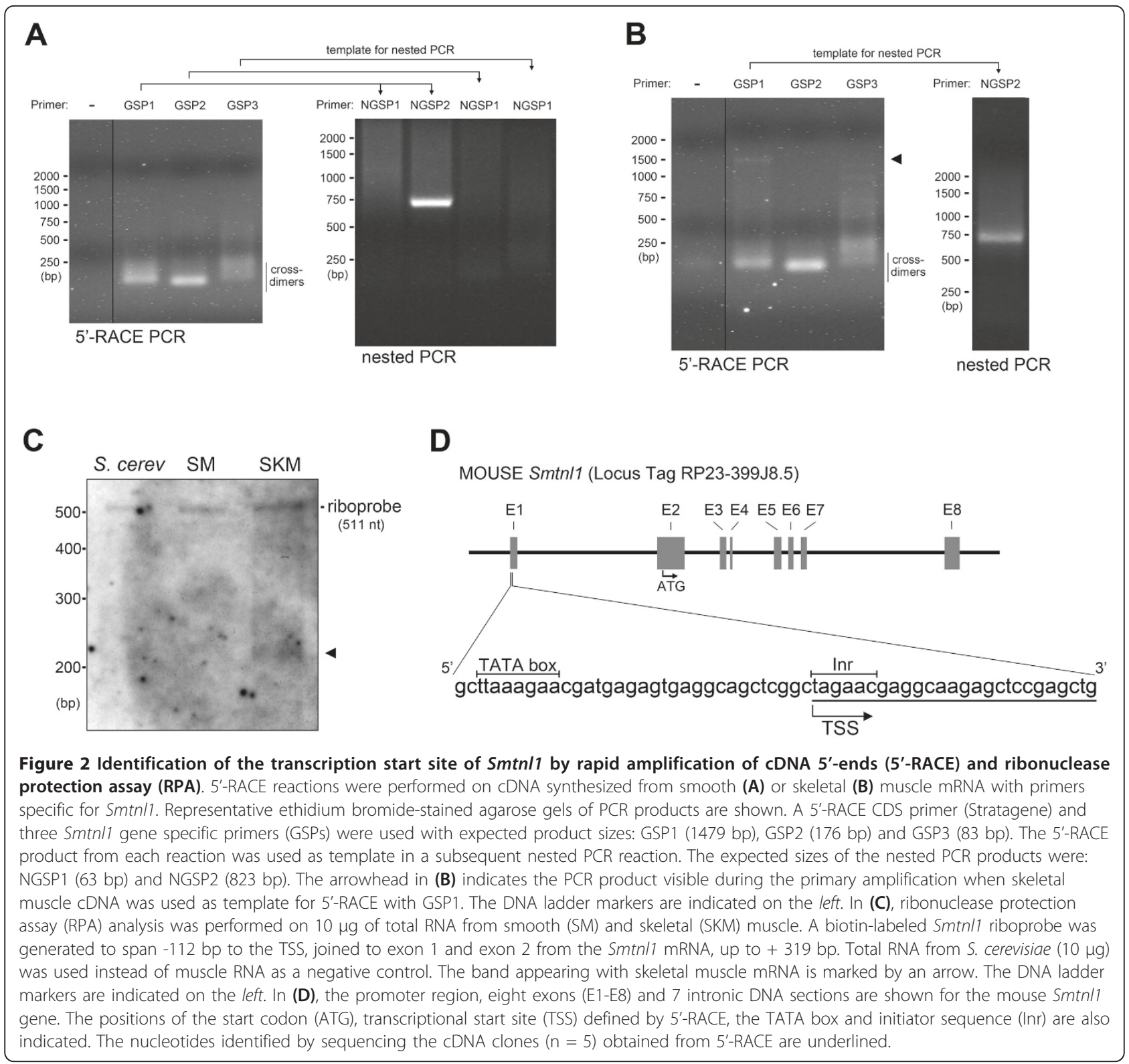

sequence that functioned as enhancer elements, -218 to -468 and 0 to -118 . Furthermore, two inhibitory elements were also defined, -1637 to -1869 and -118 to -218 . These results demonstrate that the first $118 \mathrm{bp}$ upstream of the Smtnl1 TSS could significantly enhance luciferase production, suggesting that the core promoter was located in this region. Importantly, the region of the core promoter did not include the TSS previously annotated in NCBI. Our data confirms that the region immediately upstream of the NCBI-annotated TSS could not enhance expression, which further verifies our newly identified TSS downstream of the NCBI-annotated TSS. We further investigated the sequence of the first $118 \mathrm{bp}$ upstream of the TSS for possible promoter elements.
A TTAAA motif, $31 \mathrm{nt}$ upstream of the TSS, was $100 \%$ conserved amongst all the mammalian Smtnl1 promoters investigated and is predicted to serve as a TATAbox. We were unable to identify a CAAT element.

The promoter region just upstream and including the TSS is highly conserved in mammals, emphasizing its importance. The TSS of some mammals is not annotated in this region, and this might be due to difficulties in identifying 5'-UTRs within the corresponding ESTs. Further corroborating this possibility was the distance between the TSS (equivalent to murine exon 1) and the translational start-site (ATG) in murine exon 2. In all mammals, this distance was similarly expansive (e.g., $3400 \mathrm{bp}(\mathrm{dog})$ to $5200 \mathrm{bp}(\mathrm{cow}))$. We propose that all 
A

(kb) $-3.0 \quad-2,0 \quad-1,0 \quad \underset{0}{\longrightarrow} \longrightarrow$ Smtn/1

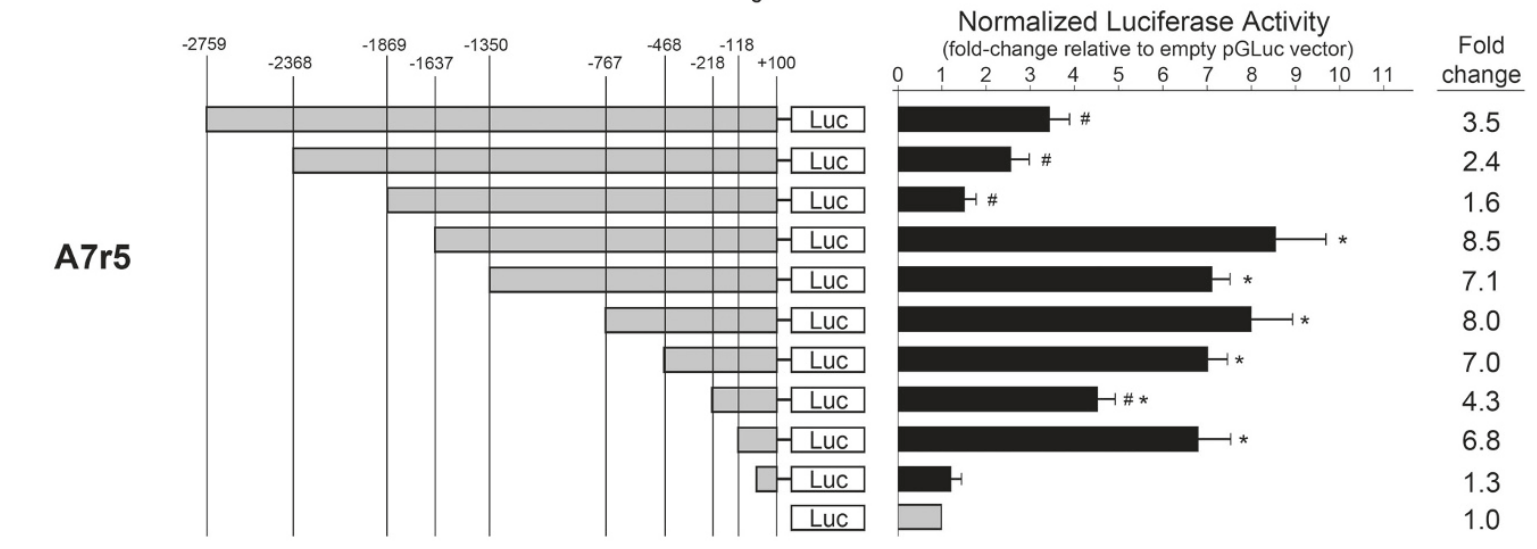

B
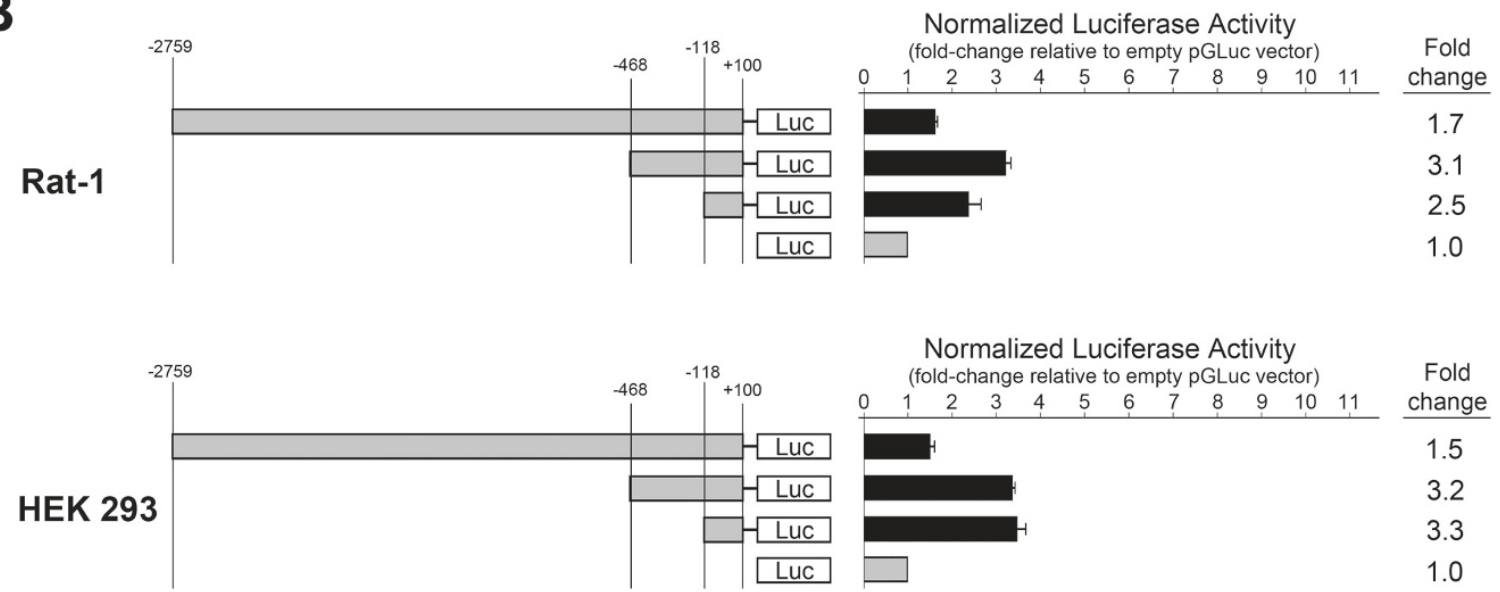

Figure 3 Identification of enhancer and repressor regions within the $\mathbf{S m t n} / 1$ promoter. In (A), various deletions (left) of the $S m t n / 1$ promoter region spanning +100 bp to -2759 bp were used to drive expression of the Gaussia luciferase gene. The positions were numbered from the transcriptional start site (TSS) identified by 5'-RACE in this study. A7r5 cells were transiently co-transfected with the pGLuc reporter and the constitutively active $\beta$-galactosidase ( $\beta$-gal) reporter vector, pbAct $\beta$-gal for normalization. After $48 \mathrm{hr}$, the media and cell lysates were assayed for luciferase and $\beta$-gal activities, respectively. Luciferase activity was normalised to $\beta$-gal activity and expressed as fold-increase (right) relative to the activity of the empty pGLuc vector. All values are mean \pm S.E.M. $(n=5-8)$. ${ }^{-}$significantly different from $+100-0$ bp promoter region; \#- significantly different from 0 to -118 bp promoter region. Statistical analysis was completed with ANOVA and Student-Neumann-Keuls post-hoc analysis, $\mathrm{p}<$ 0.05. Additional studies (B) also examined luciferase expression in Rat 1 fibroblast and human embryonic kidney (HEK 293 ) cell lines 48 hr after transient co-transfection with the pGLuc reporter vector and pbAct- $\beta$-gal for normalization. All values are mean \pm S.E.M. $(n=3)$.

mammals investigated have a TSS located downstream of the conserved region, similar to the murine promoter. This would add an additional 5'-UTR exon 1 to human, rat and horse Smtnll.

We also investigated whether the pattern of luciferase inductions observed with the Smtnl1 promoter sequences resulted from a specific set of transcription factors unique to A7r5 smooth muscle cells. For this, the activities of three Smtnl1 promoter constructs were also assessed in HEK 293 (human embryonic kidney) and Rat-1 (rat fibroblast) cell lines (Figure 3B). The same pattern of luciferase induction was seen in these cell lines; however, the maximal activation measured was only 3 -fold, a significant reduction when compared to the maximal activity found in A7r5 smooth muscle cells. The identified cis-acting promoter spanned at least $1869 \mathrm{bp}$ and could up- or down-regulate expression in A7r5, Rat-1 and HEK 293 cells, depending on which region was present. However, the much higher maximum activation in smooth muscle cells compared to fibroblast and kidney cells suggests restrictive control of Smtnl1 expression in non-muscle cells and emphasizes 
the importance of SMTNL1 expression in muscle contractile function.

\section{In silico analysis of putative transcription factor binding sites within Smtnl1}

A comparison of TSS sites in various mammalian Smtnl1 genes was undertaken, and BLAST searches revealed that exon 1 is not translated and the murine ATG start codon is located at the beginning of exon 2 . In the case of some mammals (i.e., human, rat and horse), the Smtnl1 gene starts with the ATG in exon 1 and is analogous to murine exon 2. Further investigation of these Smtnl1 variants revealed the location of a genomic region that has not been annotated. This region lays several thousand bases upstream with high similarity to the murine exon 1. Comparison of various mammalian SMTNL1 protein sequences revealed the highest conservation at the $\mathrm{N}$ - and $\mathrm{C}$-termini (aa 1 - 17 as well as aa 209 - 459). Thus, it is not surprising that, based on protein sequence and gene sequence alignment, the exon including the start codon was annotated in all mammals while the upstream exon 1 was sometimes not annotated. In agreement with the observation that exon 1 is often located far upstream of exon 2, there is approximately 3000 to 4000 bp separating the conserved region corresponding to the mouse 5'-UTR and the ATG start codon of Smtnl1 from various mammalian species (Figure 4A). Based on these findings, it is reasonable to predict that the human Smtnl1 TSS lies upstream of the region homolog to murine smtnl1 exon 1, even though it remains to be annotated. Indeed, the region just upstream of the murine TSS is highly conserved in mammals, with $>75 \%$ percent identity (Figure $4 \mathrm{~A}$ ).

The highest conservation in the murine Smtnl1 5'UTR sequence was located between $-80 \mathrm{bp}$ and $-220 \mathrm{bp}$ relative to the newly derived TSS, suggesting functional importance. Indeed, the region corresponds in part to the strongly activating construct determined with the luciferase reporter assay (i.e., +100 to -118 ). We searched for transcription factor binding sites (TFBS) in this region using the $\mathrm{PATCH}$ program that utilizes the TRANSFAC $^{\circledR}$ database of cis-acting transcription factors $[16,17]$. Possible mammalian TFBSs in this region include AP2 $\alpha$, SF-1, RXR $\alpha$, SP1, ER and c-myc amongst others (Figure 4B). Intriguingly, this region of the Smtnl1 promoter also possesses a putative TFBS for MyoD

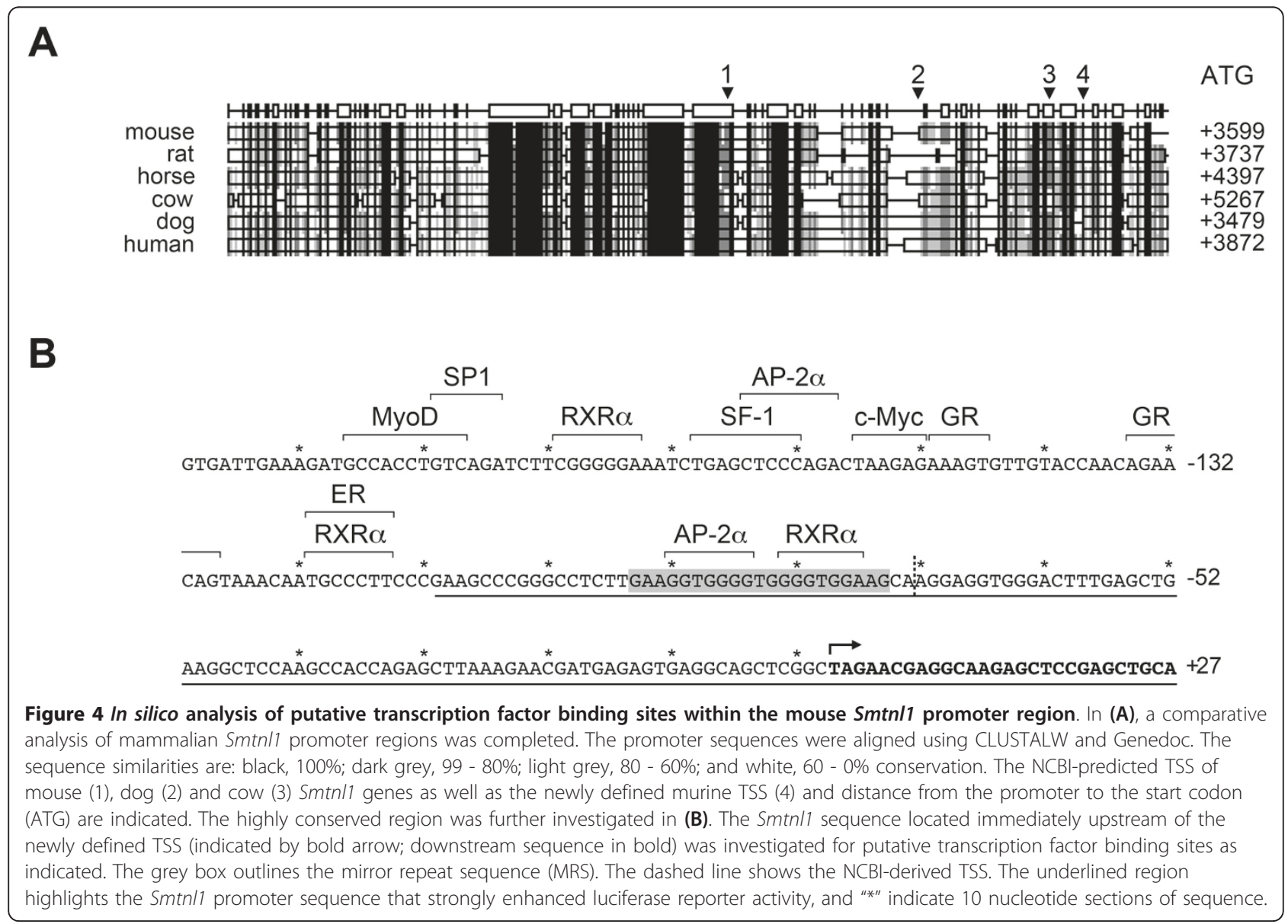


(myogenic differentiation-1), a transcriptional activator of muscle-specific genes [18], located $190 \mathrm{bp}$ upstream of the TSS. MyoD shows highest transcriptional activation when dimerized with another MyoD or other family members (e.g., Sp1, Mef2 and Pbx) [19,20]. Indeed, possible non-canonical sites for MyoD as well as Sp1 are located adjacent to the primary MyoD site in the Smtnl1 promoter. Thus, regulation of Smtnl1 expression via MyoD would explain the robust expression found in skeletal muscle when compared to other tissues. We completed EMSAs with nuclear extracts from mouse skeletal muscle and DNA probes derived from the MyoD site in the Smtnl1 promoter (Figure 5A). Gel shifts were suggestive of protein binding to the Smtnl1 MyoD sequence and could be inhibited with addition of excess unlabelled probe as a competitive inhibitor. Moreover, EMSAs completed in the presence of MyoD antibody exhibited a defined "supershifting" band.

The highest density of putative TFBSs lay within the sequence GAAGGTGGGGTGGGGTGGAAG of Smtnl1 that forms a $20 \mathrm{bp}$ mirror-repeat sequence (MRS). Mirror repeats are commonly associated with the formation of an intramolecular triple-helical conformation termed H-DNA [21,22]. Many promoters, especially those integral to genes involved in growth regulation, have $\mathrm{H}$ DNA forming mirror repeats. The Smtnl1 MRS lies within a region of high transcriptional activation as determined by luciferase reporter assay, and it is possible that this region is of paramount importance for Smtnl1 expression. EMSAs completed with MRS oligonucleotide probes and nuclear extracts from A7r5 cells demonstrated a single intense band (Figure 5B). This binding pattern was significantly altered when nuclear extracts from HEK293 cells were used; in this case, multiple bands were observed. The results are suggestive of transcription factor binding to the MRS; however, more in depth investigation will be required to identify the specific proteins involved.

Other transcription factors are of interest based on their function and our knowledge of SMTNL1. Putative binding sites for the nuclear hormone receptor steroidogenic factor-1 (SF-1) as well as estrogen receptor (ER) are present. These receptors might be responsible for the observed differences in Smtnl1 expression during sexual development in males [3] as well as during pregnancy in females [4]. RXR $\alpha$ (retinoid $\times$ receptor alpha) modulates the actions of other hormone receptors and plays a role in myogenesis. RXR $\alpha$ is also responsible for the activation of MyoD and deficiency in RXR $\alpha$ causes defects in skeletal muscle development [23]. Furthermore, RXR $\alpha$ is a binding partner for PPAR (peroxisome proliferator-activated receptor) transcription factors [24]. In turn, PPAR $\gamma$ regulates endurance-exercise induced fiber type remodeling [25]. Since endurance-exercise caused up-regulation of Smtnl1, RXR $\alpha$ together with PPAR $\gamma$ might be the responsible factors. Notable is the absence of a consensus CC-A/T rich-GG (CArG) box, the binding site for serum response factor (SRF) that is present in smooth muscle restricted genes $[26,27]$. This distinguishes Smtnl1 from the related Smtn genes and is

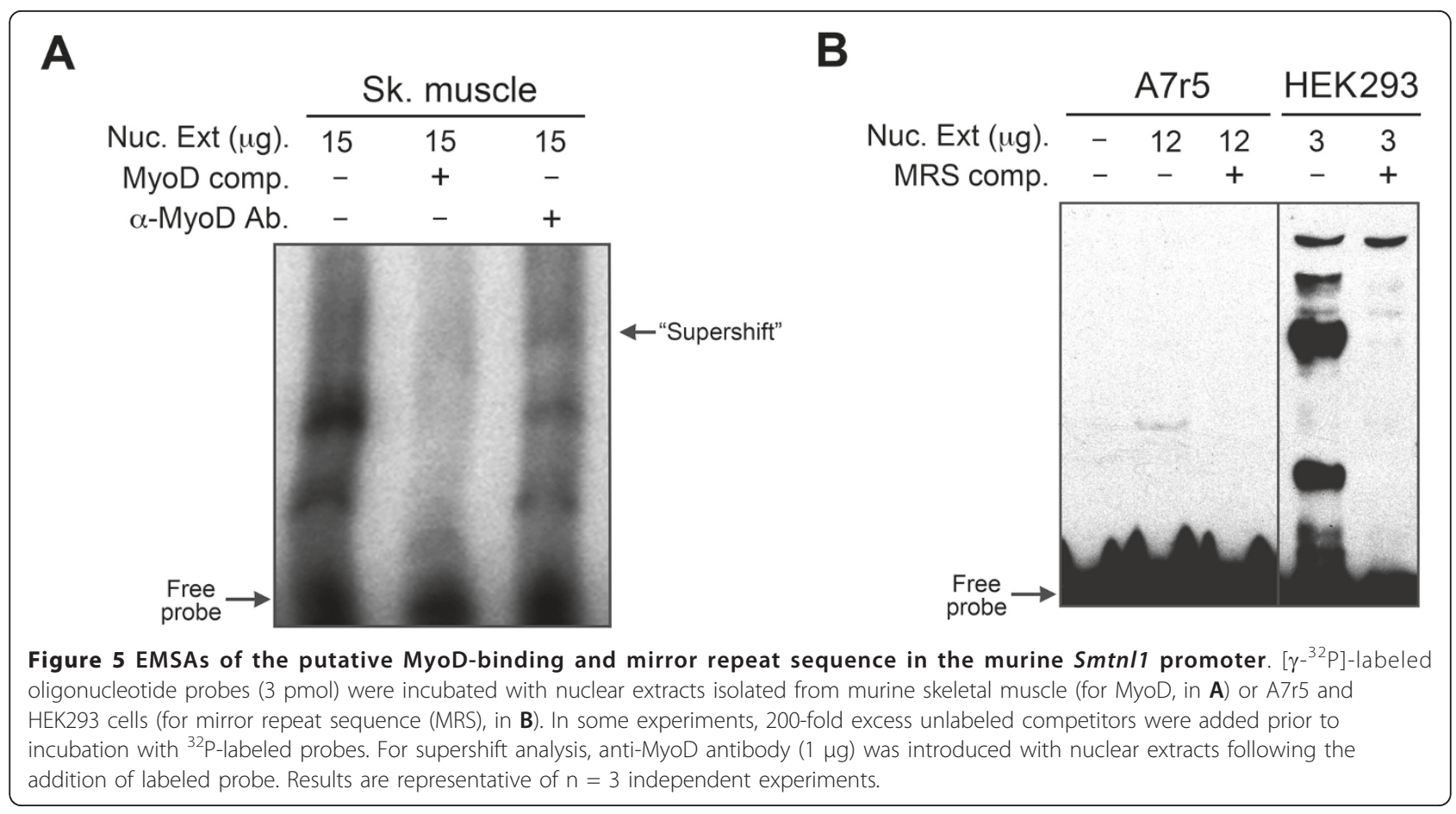


further manifested by the expression of Smtnl1 in skeletal muscle [3]. Of the numerous transcription factors predicted to bind to the proximal promoter, several could be responsible for the adaptive expression of Smtnl1 after endurance-exercise, pregnancy and sexual development in smooth and skeletal muscle. This will be subject to further research.

\section{Conclusions}

In the present study, we identified Smtnl1 expression in all types of smooth muscle tissue (e.g., phasic and tonic; vascular and visceral) examined, albeit at lower levels than found in skeletal muscle. Current evidence supports a key role for SMTNL1 in adaptive responses to physiological stresses (e.g., pregnancy, exercise and sepsis). Therefore, defining key elements of the murine Smtnl1 gene will enable better understanding of mechanisms for smooth and skeletal muscle contractile plasticity in response to physiological stresses.

\section{Methods}

\section{RNA samples and cell culture}

Mouse total skeletal muscle RNA and mouse total smooth muscle RNA (from small intestine) were purchased from Clontech Laboratories (Mountain View, CA). Rat aortic smooth muscle (A7r5, [ATCC:CRL-1444]), human embryonic kidney (HEK 293, [ATCC:CRL-1573]) and rodent fibroblast (Rat 1, [ATCC:CRL-2210]) cells were maintained in Dulbecco's Modified Eagle Medium (DMEM) supplemented with $10 \%$ fetal bovine serum (FBS) in a humidified tissue culture incubator at $37^{\circ} \mathrm{C}$ with $5 \% \mathrm{CO}_{2}$. DMEM and FBS were purchased from Invitrogen (Carlsbad, CA).

\section{Reverse transcription Polymerase Chain Reaction (RT-PCR)} For all RT-PCR reactions, the 'standard PCR' kit from Fermentas (Glen Burnie, MD) was used. All reactions were carried out using a thermal cycler (Eppendorf Mastercycler; Westbury, NY). Skeletal muscle as well as aorta, ileum and colonic smooth muscle tissues were removed from male mice $(\mathrm{C} 57 \mathrm{Bl} / 6)$ that had been anesthesized and euthanized according to protocols approved by the University of Calgary Animal Care and Use Committee. Total RNA and mRNA were extracted using the RNeasy Mini kit (Qiagen) or Ambion Micro Poly(A) Pure extraction kit, respectively. First strand $\mathrm{CDNA}$ was synthesized using the Sensiscript RT kit (Qiagen). For the PCR amplification, Phusion Taq DNA polymerase (New England Biolabs) was used with the gene-specific primers mSmtnl1-F1, mSmtnl1-R1, mSmtnl1-nested-F1 and mSmtnl1-nestedR1. The primer sequences are noted in Supplementary Table 1. Primers were selected to be unique to Smtnl1 mRNA and did not occur within the transcripts of the other smoothelin family members. The nested-RT-PCR reactions were used because they offer increased specificity and sensitivity. RT-PCR reactions for GAPDH were performed on all RNA samples to verify the integrity of the RNA.

\section{Northern Blot}

Mouse Smtnl1 cDNA (1 - 1038 bp) was radiolabeled with $\left[\alpha^{32} \mathrm{P}\right]$-dCTP by the random-priming method using a PrimeIt II Random Primer Labeling kit (Stratagene). Unincorporated nucleotide was removed with a NucAway Spin Column (Ambion). A multiple tissue Northern blot that contained poly(A)+ RNA from a variety of human tissues was purchased from Clontech. The poly (A)+ RNA membrane was prehybridized in ExpressHyb solution for $30 \mathrm{~min}$ at $68^{\circ} \mathrm{C}$. Radiolabeled cDNA probe $\left(\sim 5 \times 10^{6} \mathrm{cpm} / \mathrm{mL}\right)$ was denatured at $100^{\circ} \mathrm{C}$ for $2 \mathrm{~min}$ then rapidly chilled on ice. The membrane was incubated with the radiolabeled probe in $5 \mathrm{~mL}$ of fresh ExpressHyb with continuous shaking for $1 \mathrm{~h}$ at $68^{\circ} \mathrm{C}$. Following hybridization, the blot was washed for $30 \mathrm{~min}$ at $23^{\circ} \mathrm{C}$ with $2 \times \mathrm{SSC}(300 \mathrm{mM} \mathrm{NaCl}, 30 \mathrm{mM}$ Na citrate, $\mathrm{pH} 7.0$ ), 0.5\% SDS (several washes) and twice for 20 min with $0.1 \times$ SSC $(15 \mathrm{mM} \mathrm{NaCl}, 1.5 \mathrm{mM} \mathrm{Na}$ citrate, $\mathrm{pH} 7.0), 0.1 \% \mathrm{SDS}$ at $50^{\circ} \mathrm{C}$. The membrane was then subjected to autoradiography.

\section{Rapid Amplification of 5' cDNA ends (5'-RACE)}

The SMART RACE cDNA Amplification kit from Clontech Laboratories was used to amplify $5^{\prime}$-cDNA ends of mouse smooth and skeletal muscle mRNA. First strand cDNA synthesis was carried out according to the manufacturer's instructions with mouse total RNAs (Clonetech, $1 \mu \mathrm{g}$ ) from intestinal smooth muscle or skeletal muscle, 5' CDS primer A, SMART II A oligonucleotide and TITANIUM Taq DNA polymerase (Advantage 2 PCR System, Clontech). The 5'-RACE PCR reaction was performed according to the manufacturer's protocol using Universal Primer A mix (UPM, forward primer) and one of three Smtnl1 gene specific primers (GSP1-3; reverse primers). The procedure incorporates an additional $50 \mathrm{bp}$ to the $5^{\prime}$-end of the PCR product. To verify the RACE products, nested 5'-RACE PCR was carried out using nested UPM (NUPM) forward primer and one of three nested Smtnl1 gene specific reverse primers (NGSP1, NGSP2). The nested RACE products were separated on a $1 \%$ agarose gel, extracted and cloned into the TOPO-TA vector (Invitrogen, Carlsbad, CA). All nested RACE products were sequenced at the University of Calgary DNA core service.

\section{Ribonuclease protection assay (RPA)}

To verify the translation start site (TSS), RPA was performed on murine smooth and skeletal muscle mRNA. The template for the riboprobe was a chimera of murine 
genomic DNA, comprising the 5'-UTR and exon 1 of the $S m t n l 1$ gene $(-52 \mathrm{nt}$ to $+160 \mathrm{nt}$ relative to the NCBI-predicted TSS [Gene ID: 68678]) plus the 5'-end of exon 2 taken from Smtnl1 (+161 nt to +319 nt [GenBank: NM_024230.1]) and a T7 primer overhang. The template was generated using standard PCR methods: the BAC clone RP23-125A8 (Children's Hospital Oakland Research Institute, Oakland, CA) and primers RPA-NCS and RPA-NCA were used for the genomic portion, and the product of the 5'-RACE together with primers RPA-M1-2 S and RPA-M1-2A was used as the mRNA part. A second PCR with primers RPA-FR1 and RPA-RT7 engineered the T7 promoter into the RPAtemplate. The riboprobe was synthesized using T7 DNA dependent RNA transcriptase (New England Biolabs, Ipswich, MA) according to the manufacturer's instructions. To label the riboprobe, biotin-18-UTP/UTP (1:5 ratio) was used in the reaction mix (Invitrogen). For the RPA, the RPA III kit (Ambion, Austin, TX) was used with $10 \mu \mathrm{g}$ mRNA and $100 \mathrm{pg}$ riboprobe. Briefly, murine mRNA and the riboprobe were hybridized to form dsRNA $\left(40.5^{\circ} \mathrm{C}\right.$, overnight). Then, any remaining singlestranded mRNA and riboprobe were digested with RNase. The protected RNA was resolved by denaturing PAGE and transferred to a SensiBlot nylon membrane (Fermentas, Glen Burnie, MD). The remaining biotin18-UTP-labeled riboprobe was detected using Ambion's Brightstar Biodetect kit according to the manufacturer's instructions.

\section{Luciferase Assay}

A luciferase reporter assay was used to investigate the putative Smtnl1 promoter sites in the 5'-flanking region. The Smtnl1 gene and 5'-flanking sequence spanning bp 84662089 to bp 84651332 of mouse chromosome 2 was obtained from BAC clone RP23-125A8. Different lengths of Smtnl1 5'-flanking DNA were generated by PCR with the BAC plasmid as template and cloned into the pGLuc-Basic vector (New England Biolabs, Ipswich, MA). The antisense primer utilized for all constructs engineered a BamHI site at +100 bp downstream of the transcription start site identified by our previous 5'RACE/RPA analysis. Ten different sense primers were used, adding an EcoRI site for cloning into the pGLucBasic vector. All clones were verified by DNA sequencing. Using Metafectene-Pro (Biontex Laboratories, München, Germany) and following the manufacturer's protocols, we transiently transfected A7r5, HEK 293 or Rat-1 cells with pGLuc-Basic vectors $(5 \mu \mathrm{g})$ containing the various Smtnl1 promoter constructs and pbActb-gal vector $(1 \mu \mathrm{g})$. The pbActb-gal vector expresses $\beta$-galactosidase under the constitutively active $\beta$-actin promoter and was a kind gift from Dr. J. Cross (University of Calgary). Twenty-four hours before transfection, cells were plated in $30-\mathrm{mm}$ dishes to produce a density of $\sim 80 \%$ confluence. At 48 hours post-transfection, the media was assayed for luciferase activity using the Gaussian Luciferase Assay kit (New England Biolabs) and cell lysates were assayed for $\beta$-galactosidase activity, in quadruplicate samples of three independent experiments. To account for differences in transfection efficiency, the luciferase activity was normalized to $\beta$-galactosidase activity and expressed as \% change relative to the activity of empty pGLuc-Basic vector (defined as $100 \%$ ). As a positive control, pGLuc-CMV was used in separate transfections.

\section{Electrophoretic mobility shift assay (EMSA)}

DNA binding was assessed by EMSAs with standard procedures [28] using nuclear extracts prepared from A7r5 cells or mouse skeletal muscle tissue (hind-limb). Double-stranded oligonucleotide probes containing the mirror-repeat (MRS) and putative MyoD binding sequences located within the Smtnl1 promoter region were synthesized (sense strands: MRS: 5'-GAAGGTG GGGTGGGGTGGAAG-3'; MyoD: 5'-ATTGAAAGA TGCCACCTGTCAGATCT-3'). Each oligonucleotide was annealed to its complementary strand, end-labeled with $\left[\gamma_{-}{ }^{32} \mathrm{P}\right]$-ATP using T4 polynucleotide kinase and then purified with G-25 Sepharose mini-columns. Binding reactions were carried out in a mixture containing 1-15 $\mu \mathrm{g}$ nuclear extract proteins in $20 \mu \mathrm{L}$ of binding buffer $(10 \mathrm{mM}$ Tris, $50 \mathrm{mM} \mathrm{KCl,} 1 \mathrm{mM}$ DTT, 5\% glycerol, pH 7.5). Radiolabeled, double-stranded oligonucleotide probe $(3 \mathrm{pmol})$ was added, and mixtures were incubated at room temperature for $30 \mathrm{~min}$ prior to resolution on $5 \%$ non-denaturing polyacrylamide gels $(1 \times$ $\mathrm{TBE})$. The gels were then dried, and the radioactive bands were developed using a Storm phosphoimager (GE Healthcare). For competition experiments, unlabeled MyoD or MRS oligonucleotide competitor (200fold excess) was added to the reaction mixture before incubation with the radiolabeled probe. For MyoD supershift assays, the reaction mixtures were incubated with $1 \mu \mathrm{g}$ of anti-MyoD antibody prior to the addition of radiolabeled probe.

\section{Bioinformatic promoter analysis}

The 5'-UTR of the murine Smtnl1 was searched against mammalian genomes using the BLAST algorithm. Regions with high alignment score (expectancy value $\mathrm{E}$ $<10^{-10}$ ) were aligned using CLUSTALX and GeneDoc after visual inspection. All mammalian sequences aligning to the murine gene (i.e., bp 25704307 to 25703966 of the reverse strand of GenBank: NT-039297.7; 197 bp upstream of the TSS defined by NCBI) were selected for further investigation. The sequence identity was approximately $54 \%$. The region between +38 to -94 
relative to the NCBI TSS (-79 to -211 of the newly defined TSS) was termed highly conserved with an identity of $>72 \%$ between all sequences. This region of highly conserved sequence was analyzed with the PATCH program for putative transcription factor binding sites based on the Transfac ${ }^{\circledR}$ database $[16,17]$.

\section{List of Abbreviations}

bp: base pair; CGMP: cyclic-guanidine monophosphate; DMEM: Dulbecco's Modified Eagle Medium; EMSA: electrophoretic mobility shift assay; FBS: fetal bovine serum; MRS: mirror repeat sequence; MyoD: myogenic differentiation1; nt: nucleotide; PKA: protein kinase A; PKG: protein kinase G; $5^{\prime}$-RACE: rapid amplification of $5^{\prime}$ CDNA ends; $3^{\prime}$-RACE: rapid amplification of $3^{\prime}$ ends; RPA: ribonuclease protection assay; SMTN: smoothelin; SMTNL1: smoothelin-like 1; SRF: serum response factor; TFBS: transcription factor binding site; TSS: transcriptional start site; 5'-UTR: $5^{\prime}$ untranslated region.

\section{Acknowledgements}

This work was supported by research grants from the Canadian Institutes of Health Research (CIHR, MOP-72720) and the Heart and Stroke Foundation of Canada (HSFC). MB was recipient of a HSFC fellowship, and ST was recipient of an Alberta Innovates - Health Solutions (AlHS) Studentship. JM holds an AlHS Senior Scholar Award and Canada Research Chair in Smooth Muscle Pathophysiology.

\section{Authors' contributions}

AU-L carried out MyoD EMSAs and bioinformatic work, interpreted the data and drafted the manuscript. ST performed RT-PCR for Smtnl1 in mouse tissues. SM carried out 5' RACE, RPA and luciferase assay analyses. RW contributed to the design of 5'-RACE, RPA and nested RT-PCR experiments as well as the interpretation of the data. MB performed the northern blot. JM conceived and designed the study, interpreted the data and revised the manuscript for intellectual content. All authors read and approved the final manuscript.

Received: 20 October 2010 Accepted: 27 February 2011 Published: 27 February 2011

\section{References}

1. Borman MA, MacDonald JA, Haystead TA: Modulation of smooth muscle contractility by CHASM, a novel member of the smoothelin family of proteins. FEBS Lett 2004, 573:207-13.

2. Borman MA, Freed TA, Haystead TA, MacDonald JA: The role of the calponin homology domain of smoothelin-like 1 (SMTNL1) in myosin phosphatase inhibition and smooth muscle contraction. Mol Cell Biochem 2009, 327:93-100.

3. Wooldridge AA, Fortner CN, Lontay B, Akimoto T, Neppl RL, Facemire C, Datto MB, Kwon A, McCook E, Li P, Wang S, Thresher RJ, Miller SE, Perriard JC, Gavin TP, Hickner RC, Coffman TM, Somlyo AV, Yan Z, Haystead TA: Deletion of the protein kinase A/protein kinase G target SMTNL1 promotes an exercise-adapted phenotype in vascular smooth muscle. J Biol Chem 2008, 283:11850-9.

4. Lontay B, Bodoor K, Weitzel DH, Loiselle D, Fortner C, Lengyel S, Zheng D, Devente J, Hickner R, Haystead TA: Smoothelin Like 1 protein regulates myosin phosphatase targeting subunit 1 expression during sexual development and pregnancy. J Biol Chem 2010, 285:29357-29366.

5. Ulke-Lemee A, Ishida H, Borman MA, Valderrama A, Vogel HJ, MacDonald JA: Tropomyosin-binding properties of the CHASM protein are dependent upon its calponin homology domain. FEBS Lett 2010, 584:3311-3316.

6. Ishida H, Borman MA, Ostrander J, Vogel HJ, MacDonald JA: Solution structure of the calponin homology $(\mathrm{CH})$ domain from the smoothelinlike 1 protein: a unique apocalmodulin-binding mode and the possible role of the C-terminal type-2 $\mathrm{CH}$-domain in smooth muscle relaxation. $J$ Biol Chem 2008, 283:20569-78.

7. van der Loop FT, Gabbiani G, Kohnen G, Ramaekers FC, van Eys GJ: Differentiation of smooth muscle cells in human blood vessels as defined by smoothelin, a novel marker for the contractile phenotype. Arterioscler Thromb Vasc Biol 1997, 17:665-71.
8. Georgijevic S, Subramanian Y, Rollins EL, Starovic-Subota O, Tang AC, Childs SJ: Spatiotemporal expression of smooth muscle markers in developing zebrafish gut. Dev Dyn 2007, 236:1623-32.

9. Rensen SS, Niessen PM, van Deursen JM, Janssen BJ, Heijman E, Hermeling E, Meens M, Lie N, Gijbels MJ, Strijkers GJ, Doevendans PA, Hofker MH, De Mey JG, van Eys GJ: Smoothelin-B deficiency results in reduced arterial contractility, hypertension, and cardiac hypertrophy in mice. Circulation 2008, 118:828-36.

10. Niessen $P$, Rensen $S$, van Deursen J, De Man J, De Laet A, Vanderwinden JM, Wedel T, Baker D, Doevendans P, Hofker M, Gijbels M, van Eys G: Smoothelin-a is essential for functional intestinal smooth muscle contractility in mice. Gastroenterology 2005, 129:1592-601.

11. Rensen SS, Thijssen VL, De Vries CJ, Doevendans PA, Detera-Wadleigh SD, Van Eys GJ: Expression of the smoothelin gene is mediated by alternative promoters. Cardiovasc Res 2002, 55:850-63.

12. Rensen SS, Niessen PM, Long X, Doevendans PA, Miano JM, van Eys GJ: Contribution of serum response factor and myocardin to transcriptional regulation of smoothelins. Cardiovasc Res 2006, 70:136-45.

13. Wang D, Chang PS, Wang Z, Sutherland L, Richardson JA, Small E, Krieg PA, Olson EN: Activation of cardiac gene expression by myocardin, a transcriptional cofactor for serum response factor. Cell 2001, 105:851-62.

14. Chen J, Kitchen CM, Streb JW, Miano JM: Myocardin: a component of a molecular switch for smooth muscle differentiation,. J Mol Cell Cardiol 2002, 34:1345-56.

15. Kramer J, Quensel C, Meding J, Cardoso MC, Leonhardt H: Identification and characterization of novel smoothelin isoforms in vascular smooth muscle. J Vasc Res 2001, 38:120-32.

16. Matys V, Kel-Margoulis OV, Fricke E, Liebich I, Land S, Barre-Dirrie A, Reuter I, Chekmenev D, Krull M, Hornischer K, Voss N, Stegmaier P, LewickiPotapov B, Saxel H, Kel AE, Wingender E: TRANSFAC and its module TRANSCompel: transcriptional gene regulation in eukaryotes. Nucleic Acids Res 2006, 34:D108-10.

17. Gene-regulation.com. [http://www.gene-regulation.com].

18. Weintraub H, Tapscott SJ, Davis RL, Thayer MJ, Adam MA, Lassar AB, Miller AD: Activation of muscle-specific genes in pigment, nerve, fat, liver, and fibroblast cell lines by forced expression of MyoD. Proc Natl Acad Sci USA 1989, 86:5434-8.

19. Weintraub H, Davis R, Lockshon D, Lassar A: MyoD binds cooperatively to two sites in a target enhancer sequence: occupancy of two sites is required for activation. Proc Natl Acad Sci USA 1990, 87:5623-7.

20. Knoepfler PS, Bergstrom DA, Uetsuki T, Dac-Korytko I, Sun YH, Wright WE, Tapscott SJ, Kamps MP: A conserved motif N-terminal to the DNA-binding domains of myogenic bHLH transcription factors mediates cooperative DNA binding with pbx-Meis1/Prep1. Nucleic Acids Res 1999, 27:3752-61.

21. Mirkin SM, Lyamichev VI, Drushlyak KN, Dobrynin VN, Filippov SA, FrankKamenetskii MD: DNA H form requires a homopurine-homopyrimidine mirror repeat. Nature 1987, 330:495-7.

22. Htun H, Dahlberg JE: Single strands, triple strands, and kinks in H-DNA. Science 1988, 241:1791-6.

23. Szanto A, Narkar V, Shen Q, Uray IP, Davies PJ, Nagy L: Retinoid $\times$ receptors: X-ploring their (patho)physiological functions. Cell Death Differ 2004, 11(Suppl 2):S126-43.

24. Chan LS, Wells RA: Cross-talk between PPARs and the partners of RXR: a molecular perspective.. PPAR Res 2009, 925309.

25. Luquet S, Lopez-Soriano J, Holst D, Fredenrich A, Melki J, Rassoulzadegan M, Grimaldi PA: Peroxisome proliferator-activated receptor delta controls muscle development and oxidative capability. FASEB J 2003, 17:2299-301.

26. $M c D o n a l d ~ O G$, Owens GK: Programming smooth muscle plasticity with chromatin dynamics. Circ Res 2007, 100:1428-1441.

27. Miano JM, Long X, Fujiwara K: Serum response factor: master regulator of the actin cytoskeleton and contractile apparatus. Am J Physiol Cell Physiol 2007, 292:C70-C81.

28. Noh OJ, Park YH, Chung YW, Kim IY: Transcriptional regulation of selenoprotein $\mathrm{W}$ by MyoD during early skeletal muscle differentiation. J Biol Chem 2010, 285:40496-40507.

doi:10.1186/1471-2199-12-10

Cite this article as: Ulke-Lemée et al:: Mapping and functional characterization of the murine Smoothelin-like 1 promoter. BMC Molecular Biology 2011 12:10. 